EGU2020-9546

https://doi.org/10.5194/egusphere-egu2020-9546

EGU General Assembly 2020

(c) Author(s) 2020. This work is distributed under

the Creative Commons Attribution 4.0 License.

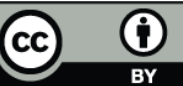

\title{
Influence of Aeolus data assimilation on the representation of gravity waves in ECMWF analysis fields
}

\author{
Isabell Krisch ${ }^{1}$, Michael Rennie ${ }^{2}$, Bernd Kaifler ${ }^{1}$, Sonja Gisinger ${ }^{1}$, Oliver Reitebuch ${ }^{1}$, and Markus \\ Rapp $^{1}$ \\ ${ }^{1}$ Deutsches Zentrum für Luft- und Raumfahrt, Institut für Physik der Atmosphäre, Oberpfaffenhofen, Germany \\ ${ }^{2}$ European Centre for Medium-Range Weather Forecasts, Reading, UK
}

In January 2020, the European Centre for Medium-Range Weather Forecast (ECMWF) became the first numerical weather prediction (NWP) centre to assimilate wind observations from the new European Space Agency (ESA)'s Earth Explorer satellite Aeolus for operational forecasting. Aeolus was launched into space on August $22^{\text {nd }}, 2018$, carrying the world's first spaceborne wind lidar, the Atmospheric Laser Doppler Instrument (ALADIN). ALADIN measures profiles of line-of-sight wind components from $30 \mathrm{~km}$ altitude down to the Earth's surface or to the level where the lidar signal is attenuated by optically thick clouds.

Impact assessment studies performed at ECMWF in 2019, show improved weather forecasting skills, by assimilating Aeolus wind measurements. As a side effect, these impact experiments also reveal an influence of Aeolus data assimilation on the representation of resolved gravity waves in the ECMWF model fields. Both, orographic and non-orographic gravity waves are impacted by the Aeolus data assimilation.

This impact of Aeolus data assimilation on the representation of gravity waves in ECMWF will be presented for selected case studies in the southern hemisphere. Ground-based and airborne measurement data from the SOUTHTRAC campaign will be used for validation where available. 